\title{
Kann auf Zwangsmedikation in der klinischen Praxis verzichtet werden? - Kontra
}

\author{
Could Psychiatry Dispense with Involuntary Medication? - Contra
}

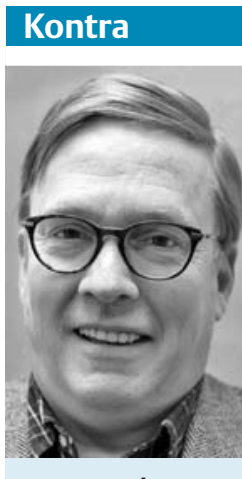

Eric Noorthoorn
Zwischen Juni 2012

und Februar 2013

war in Baden-Württemberg eine Zwangsmedikation nach den Urteilen des Bundesverfassungsgericht und des Bundesgerichtshofs nicht genehmigungsfähig. An einigen Krankenhäuern ließen sich die Folgen dieser Rechtslage studieren. Es zeigte sich eine signifikante Steigerung aggressiver Vorfälle [1]. Zu Recht wird allerdings kritisiert, dass Alternativen zu einer Zwangsmedikation in der Praxis zu wenig Beachtung finden [2]. Es ist durchaus lohnend, von den Erfahrungen anderer europäischer Länder zu lernen. Eine kürzlich publizierte Studie zeigte, dass die Unterschiede zwischen den Ländern geringer sind als die Unterschiede innerhalb eines Landes [3]. Allerdings haben sich auch unterschiedliche Kulturen herausgebildet, welche Formen von Zwangsmaßnahmen bevorzugt angewendet werden, wenn solche unumgänglich erscheinen [4]. Für die aktuelle Debatte in Deutschland geben die Erfahrungen aus den Niederlanden einen Einblick, welche Folgen sich aus einer zu restriktiven Handhabung von Zwangsmedikation ergeben können.

Ähnlich wie in Deutschland 2013 wurde in den Niederlanden bereits 1994 ein Gesetz verabschiedet, das die Verabreichung antipsychotischer Medikamente unter Zwang nur nach der gutachterlichen Stellungnahme eines zweiten, nicht in die Behandlung einbezogenen Psychiaters zuließ. Diese ausführlich zu dokumentierenden Prozeduren führen dazu, dass es viele Tage und in Einzelfällen auch Wochen dauern kann, bevor Medikamente gegen den Willen eines Patienten gegeben werden können. Nur im Notfall können Medikamente unter Zwang verabreicht werden. Oftmals stehen Psychiater vor dem ethischen Dilemma, nicht immer im Interesse des Patienten handeln zu können. Die Schwelle, wann ein Eingreifen im Falle aggressiven Eskalationen für erforderlich gehalten wird, kann zwischen den Psychiatern stark variieren.

In jedem Fall ist der Psychiater verpflichtet, den Prinzipien von Subsidiarität, Verhältnismäßigkeit und Zweckmäßigkeit zu folgen. Subsidiarität bedeutet, dass eine eingreifender Maßnahme nicht zugelassen ist, wenn eine weniger eingreifende genügt. Verhältnismäßigkeit beinhaltet das Verhältnis der Maßnahme zum begründenden Anlass, Zweckmäßigkeit, dass die Maßnahme genügend effektiv sein muss. Der gesetzlich auf 3 Monate begrenzte Einsatz von Zwangsmedikation hat dann das Ziel, eine stagnierende Behandlung zu unterstützen und eine Entlassung mit ambulanter Folgebehandlung zu ermöglichen.

Aufgrund verschiedener ernsthafter Zwischenfälle mit Patienten, die häufig große Aufmerksamkeiten in den Medien fanden, aber auch aufgrund einer zunehmend eindeutigen Meinungsbildung in der Fachgesellschaft stellte die niederländische Regierung zwischen 2006 und 201235 Millionen Euro über die niederländische Gesundheitsbehörde an psychiatrische Krankenhäuser zur Verfügung, um Zwangsmaßnahmen jährlich um 10\% zu reduzieren. Während in den ersten Jahren die meisten geförderten Initiativen auf eine Veränderung der Verfahren im Umgang mit aggressivem Verhalten und eine Änderung der Arbeitskultur auf psychiatrischen Intensivstationen zielten, gibt es seit 2012 eine zunehmend stärkere Bewegung hin zur Entwicklung einer evidenzbasierten Praxis [5, 6]. Wie in den angelsächsischen Ländern entwickeln auch in den Niederlanden die meisten psychiatrischen Krankenhäuser Intensivstationen weiter. Um die Effekte dieser Entwicklungen $\mathrm{zu}$ bewerten, wurde seit 2007 ein landesweites Register für Zwangsmaßnahmen eingerichtet, zunächst auf freiwilliger Basis für die Kran- kenhäuser, seit 2012 verpflichtend. Dadurch wurde es möglich, die Anwendung von Zwangsmaßnahmen der verschiedenen Art in niederländischen Kliniken zunehmend repräsentativ zu beurteilen. - Tab. 1 zeigt die Daten für Isolierung und Zwangsmedikation (Fixierungen kommen in den Niederlanden kaum vor). Die Tabelle zeigt eine klare Abnahme des Anteils, vorwiegend aber auch der Dauer der Isolierungen bei einer gleichzeitigen Zunahme der Zwangsmedikation zwischen 2008 und 2011. Zwischen 2012 und 2014 ist zunächst eine weitere Senkung, später aber wieder ein leichter Anstieg des von Isolierungen betroffenen Anteils der Patienten zu sehen, während der Anteil von Zwangsmedikation betroffener Patienten in etwa konstant bleibt. Die seit 2011 eingetretene leichte Verringerung von Zwangsmedikationen ist vor dem Hintergrund der wachsenden Zahl von Krankenhäusern zu sehen, die psychiatrische Intensivstationen mit Intensivpflege eingerichtet haben sowie eine evidenzbegründete Praxis einführen. Durchschnittlich sind die Änderungen aber geringer als von der Politik erhofft. Bei der Bewertung dieser Daten sind die relativen Anteile der unterschiedlichen Maßnahmen zu berücksichtigen. Verglichen mit anderen Ländern sind die Häufigkeiten von Isolierungen ähnlich, die Dauer war aber in den Niederlanden zunächst 10mal so hoch $[7,8]$.

Zwangsmedikation wurde in den Niederlanden zwischen 2011 und 2014 nur bei $8,6-9,7 \%$ der Isolierungen verwendet, 40-50\% der Verabreichungen von Zwangsmedikationen erfolgten aber während einer Isolierung. Die durchschnittliche Dauer einer Isolierung sank um mehr als die Hälfte, wenn sie mit Zwangsmedikation kombiniert war. Über die verschiedenen Jahre ist festzustellen, dass die Unterschiede zwischen den Krankenhäusern etwa einen Faktor 10 betragen, sowohl in der Anzahl von Zwangsmedikationen als auch in der Anzahl von Isolierungen. Verschiedene Studien zeigen, dass auf der 
Tab. 1 Zahl der Krankenhäuser, Abteilungen, Isolierungen und Zwangsmedikation im Argus Register.

\begin{tabular}{|c|c|c|c|c|c|c|c|c|c|}
\hline Jahr & $\begin{array}{l}\text { Einzugs- } \\
\text { gebiet }\end{array}$ & $\begin{array}{l}\text { Kranken- } \\
\text { häuser }\end{array}$ & $\begin{array}{l}\text { Fachabtei- } \\
\text { lungen }\end{array}$ & Aufnahmen & $\begin{array}{l}\text { Isolierun- } \\
\text { gen }\end{array}$ & $\begin{array}{l}\text { Zwangs- } \\
\text { medikation }\end{array}$ & $\begin{array}{l}\text { von Isolie- } \\
\text { rung be- } \\
\text { troffen }\end{array}$ & $\begin{array}{l}\text { von Zwangs- } \\
\text { medikation } \\
\text { betroffen }\end{array}$ & $\begin{array}{l}\text { mittlere } \\
\text { Dauer von } \\
\text { Isolierungen }\end{array}$ \\
\hline & \% Holland & $\mathbf{N}$ & $\mathbf{N}$ & $\mathbf{N}$ & N & $\mathbf{N}$ & $\%$ & $\%$ & Stunden \\
\hline 2008 & 36 & 8 & 68 & 11300 & 3685 & 879 & 11,8 & 1,5 & 92 \\
\hline 2009 & 53 & 14 & 198 & 21500 & 4750 & 1067 & 10,8 & 1,6 & 43 \\
\hline 2010 & 67 & 17 & 227 & 26686 & 5525 & 2226 & 10,2 & 2,2 & 38 \\
\hline 2011 & 94 & 38 & 589 & 42960 & 7476 & 3308 & 8,7 & 2,6 & 35 \\
\hline 2012 & 99 & 55 & 1772 & 70664 & 9469 & 3354 & 6,5 & 1,8 & 17 \\
\hline 2013 & 100 & 62 & 1824 & 59098 & 10551 & 2324 & 7,9 & 2,1 & 16 \\
\hline 2014 & 100 & 74 & 1857 & 60531 & 9136 & 2384 & 7,1 & 2,1 & 14 \\
\hline \multicolumn{7}{|c|}{ durchschnittliche Änderung pro Jahr zwischen 2008 und 2014} & $-9 \%$ & $+40 \%$ & $-30 \%$ \\
\hline \multicolumn{7}{|c|}{ durchschnittliche Änderung pro Jahr zwischen 2011 und 2014} & $-8 \%$ & $-20 \%$ & $-32 \%$ \\
\hline
\end{tabular}

Ebene der einzelnen Krankenhäuser eine höhere Zahl von Isolierungen mit einer geringeren Zahl von Zwangsmedikationen hoch signifikant korreliert.

Aufgrund der vorliegenden Bewertungen des Gesetzes zur Aufnahme in psychiatrischen Kliniken (1997, 2002, 2007) und aufgrund der jetzt vorliegenden wissenschaftlichen Evaluationen wird nun ein neues Gesetz vorbereitet, das Gesetz zur verpflichtenden psychiatrischen Behandlung. Dieses Gesetz schafft die Möglichkeit, Patienten bei Gefahren für sich selbst oder andere unabhängig von der Aufnahme in einem Krankenhaus zu verpflichten, eine empfohlene Behandlung zu akzeptieren. Mit diesem Gesetz geht die niederländische Regierung in Richtung ambulante Zwangsbehandlung und bewegt sich damit in eine ähnliche Richtung wie Großbritannien. Die Überzeugung, dass die Befähigung der Patienten zur Teilnahme am sozialen Leben ein wichtiger Aspekt einer guten psychiatrischen Behandlung ist, war unter anderem ausschlaggebend, um Zwangsmedikation nicht nur wie jetzt stationär, sondern zukünftig auch ambulant einsetzen zu können.

Die aktuelle Debatte in Deutschland kann von der niederländischen Entwicklung über die letzten 20 Jahre lernen, dass ein Verbot einer Zwangsmaßnahme einer Zunahme anderer Zwangsmaßnahmen hervorbringt. Während die Gesetze in den
Niederlanden sich ändern in die Richtung, die in Deutschland bis 2011 üblich war, bewegen sich die deutschen Gesetze in die Richtung, die die Niederlande wieder verlassen haben. Bei der Gesetzgebung empfiehlt es sich, aus der Erfahrung anderer Länder zu lernen.

Danksagung: Der Autor dankt Stephan Gemsa und Marlies van der Plas für die Kommentare zur Interpretation des Gesetzes. Steven Boonzajer Flaes danken wir für seine Hilfe bei der Kalkulation der Daten im Jahren 2013 und 2014.

\section{Literatur}

1 Flammer E, Steinert T. Auswirkungen der vorübergehend fehlenden Rechtsgrundlage für Zwangsbehandlungen auf die Häufigkeit aggressiver Vorfälle und freiheitseinschränkender mechanischer Zwangsmaßnahmen bei Patienten mit psychotischen Störungen. Psychiat Prax 2015; 42: 260-266

2 Zinkler $M$. Neuregelung von Zwang - ein Auftrag für die Fachgesellschaft? Psychiat Prax 2013; 40: 115-116

3 Steinert $T$, Lepping $P$. Legal provisions and practice in the management of violent patients. A case vignette study in 16 European countries. Eur Psychiatry 2009; 24: $135-$ 141

4 Lepping P, Massood B, Flammer E et al. Comparison of restraint data from four countries. Soc Psychiatry Psychiatr Epidemiol (in press)

5 Geneeskundige Inspectie voor de Geestelijke Volksgezondheid. Wet bijzondere opnemingen in psychiatrische ziekenhuizen: BOPZ in de praktijk. Rijswijk: GIGV; 1994
6 Mierlo T, Bovenberg F, Voskes $Y$ et al. Werkboek HIC. High en intensive care in de psychiatrie. [Working book intensive care: High and intensive care in psychiatry]. Utrecht: De Tijdstroom; 2013

7 Bowers L, Alexander J, Bilgin $H$ et al. Safewards: the empirical basis of the model and a critical appraisal. J Psychiatr Ment Health Nurs 2014; 21: 354-364

8 Noorthoorn E, Lepping , Janssen $W$ et al. One-year incidence and prevalence of seclusion: Dutch findings in an international perspective. Soc Psychiatry Psychiatr Epidemiol 2015: Epub ahead of print

Sie haben eine eigene Meinung zu diesem Thema? Dann schreiben Sie uns an: psychiat-praxis@thieme.de!

Korrespondenzadresse Dr. Eric Noorthoorn

GGnet Kliniken Kreis Gelderland-Achterhoek Vordenseweg 12

7231 PA Warnsveld, Holland

Forschungsleiter am Holländischen Informationszentrum Zwangsmaßnahmen, Stichting Benchmark GGZ

Rembrantlaan 46

3723 BK Bilthoven

e.noorthoorn@ggnet.nl

Bibliografie

Dol http://dx.doi.org/

$10.1055 / \mathrm{s}-0042-105550$

Psychiat Prax 2016; 43: 189-190

(c) Georg Thieme Verlag KG

Stuttgart · New York

ISSN 0303-4259 